\title{
Fetal Surgery for Spina Bifida
}

\author{
Sacco $A^{1^{*}}$, Thompson D N P2, Ushakov F ${ }^{1}$, David $\mathrm{AL}^{1,3}$, Deprest $\mathrm{J}^{1,3}$ \\ ${ }^{1}$ Elizabeth Garrett Anderson Institute for Women's Health, University College London, UK \\ 2 Department of Paediatric Neurosurgery, Great Ormond Street Hospital for Children NHS Foundation \\ Trust, London, UK \\ ${ }^{3}$ Department of Obstetrics and Gynaecology, UZ Leuven, and Development and Regeneration, \\ Cluster Woman and Child, Biomedical Sciences, KU Leuven, Leuven, Belgium \\ *a.sacco@ucl.ac.uk
}

Words: $\quad 1498$

Figures: 4

Tables: 2

Acknowledgements:

This research is funded by the Wellcome Trust (WT101957) and Engineering and Physical Sciences Research Council (ESPRC) (NS/A000027/1). JD is also funded by the Great Ormond Street Hospital Children's Charity Fund. ALD is supported by the National Institute for Health Research University College London Hospitals Biomedical Research Centre.

Keywords:

Spina bifida, fetal surgery, fetoscopic surgery 


\section{Abstract}

Open spina bifida is often diagnosed during pregnancy. In the last 20 years, fetal repair has been shown to have benefits to the neonate and child, and is now widely available including within the UK. This article briefly examines the background, evidence, benefits and risks of fetal surgery for spina bifida. 
Introduction

Spina bifida is a neurological condition caused by incomplete closure of the neural tube in the first four weeks of gestation, leading to a defect in the bony spine through which the spinal cord is exposed. In the UK it is estimated that 700 to 900 pregnancies a year are affected. Types of spina bifida are shown in Figure 1; for this article we will focus on open spina bifida (spina bifida aperta) only.

Clinically, spina bifida leads to difficulties with mobility which is largely dependent on the level of the lesion. Sensory deficits and orthopaedic abnormalities such as talipes (clubfoot), kyphosis and scoliosis can occur as well. Most patients with spina bifida experience difficulties with bladder and bowel control. Brain changes develop, largely as a result of leakage of cerebrospinal fluid through the spinal lesion, leading to hindbrain herniation (the Arnold-Chiari or Chiari II malformation).Pathways for the circulation and absorption of CSF are underdeveloped resulting in ventricular enlargement. Postnatally progressive, ventricular enlargement (hydrocephalus) can be managed with a ventriculoperitoneal shunt; shuntsmay impart an additional burden of morbidity due to infection or blockage.. Whilst intelligence quotient (IQ) falls into the normal range for many adults with spina bifida, shunts and their complications are associated with a reduced IQ. 
Prevention and diagnosis

Spina bifida is a multi-factorial disease; increased rates have been observed in women taking anticonvulsants, those who have diabetes and/or are obese. In 1991 the Medical Research Council Vitamin Study demonstrated that periconceptual folic acid supplementation reduced the rate of spina bifida by approximately $70 \%$ in high-risk women.

Spina bifida is usually detected in pregnancy during the second trimester (anomaly) ultrasound scan at $18^{+0}$ to $20^{+6}$ weeks of gestation (Figure 2). The typical intracranial appearances include 'lemon'-shaped skull and a 'banana'-shaped cerebellum reflecting decreased calavarial growth and Chiari II malformation respectively. In the UK, the Fetal Anomaly Screening Programme advises a minimum standard of $90 \%$ for ultrasound detection of spina bifida.

\section{Standard management}

Following an antenatal diagnosis of spina bifida, $75-90 \%$ of women and couples choose termination of pregnancy in countries where there is access to legal termination of pregnancy, including the UK.

For ongoing pregnancies, the standard treatment option is postnatal surgery to close the defect, protect the spinal cord and prevent ascending infection, and is typically performed within the first two days of life. A multi-layer technique is used comprising dissection and untethering of the neural placode, followed by closure of the dura, myofascia and skin (Figure 3). Delivery of babies planned for postnatal spina bifida 
repair is usually by caesarean section, although it is unclear whether this confers a benefit over vaginal delivery.

Fetal repair of spina bifida

With ultrasound follow-up during pregnancy, a deterioration of motor function, hindbrain herniation and ventriculomegaly is often seen in fetuses with spina bifida. Animal models of spina bifida also show worsening of the condition throughout gestation. These observations led to the development of a "two-hit" hypothesis, whereby the final neurological deficit results from a combination of the primary failure of neural tube formation and secondary injury from trauma and amniotic fluid toxicity as well as persisting CSF leakage throughout pregnancy.

The first attempt at fetal repair of spina bifida in humans was in 1994 using a fetoscopic approach. This was abandoned because of high loss rates and maternal complications. The first open fetal repair was in 1998. A number of case series, primarily in the USA followed, and by 2003 over 200 fetuses had undergone open fetal repair of spina bifida with generally encouraging results. A multi-centre, randomised control clinical trial (the "MOMS" trial) comparing outcomes after fetal and postnatal surgery began in 2003 and was published in 2011. In this trial, a standardised open hysterotomy and three layered fetal spina bifida repair was performed (Figure 3) and strict inclusion/ exclusion criteria were agreed (Table 1). Women undergoing open fetal repair of spina bifida were required to agree to a caesarean birth in the current and all future pregnancies due to the increased risk of uterine rupture with an upper segment incision. 
The main outcomes of the MOMS trial (full cohort) are shown in Table 2. The rate of ventriculoperitoneal shunt placement was significantly lower in the fetal surgery group compared to the postnatal surgery group (44\% vs $82 \%$ ). Hindbrain herniation at 12 months of age was significantly reduced (64\% vs $95 \%$ postnatal surgery group); babies born after fetal surgery had a higher ability to walk independently at 30 months (42\% vs $21 \%$ postnatal surgery group).

As well as the benefits above, there were complications involved in fetal surgery (Table 2). Prematurity was shown to be a clear complication of fetal surgery; the average gestational age at birth was 34.1 weeks' gestation in the fetal surgery group and 37.3 weeks' gestation in the postnatal surgery group, and $13 \%$ of the prenatal surgery group delivered before 30 weeks' gestation.

Following the MOMS trial report, several non-randomised studies and a systematic review have been published, generally showing similar short-term outcomes. 
Long-term follow-up following fetal repair

Long term improved urologic outcomes have been reported; the more comprehensive "MOMS 2" long-term follow up study is expected to report shortly. Longer-term evidence is currently limited to case cohorts studies performed prior to the MOMS trial. Five and ten-year follow up of such cases have shown a continued benefit of reduced ventriculoperitoneal shunt rates and improved mobility; urological outcomes are more mixed but suggest better bladder function and continence in cases repaired prenatally.

For the mother, follow up at 3-14 years after open fetal surgery for spina bifida and other conditions showed that approximately half had experienced a further pregnancy, of which $20 \%$ had a miscarriage, with a preterm birth rate of approximately $20 \%$. With regards to the uterus, a dehiscence rate of $11 \%$ and a uterine rupture rate of $6 \%$ in subsequent pregnancies was found.

Fetoscopic repair of spina bifida

Largely due to the maternal morbidity of open fetal surgery, there has been extensive interest in using a fetoscopic approach to repair the fetal defect. Although the first fetal spina bifida repairs were via multiport fetoscopy, the high feto-maternal complication rate led this route to be temporarily abandoned. Unlike the standardised fetal multilayer neurosurgical repair, which is based on conventional postnatal neurosurgical repair, there is no single agreed technique for fetoscopic repair. Practice varies in terms of maternal abdominal entry (fully percutaneous or laparotomy with exteriorized uterus), uterine entry (port numbers and sizes), the neurosurgical repair 
technique itself (Figure 4), and more recently the gestational age at which the procedure is done. Fetoscopy is also controversial because, experimentally, uterine insufflation with $\mathrm{CO}_{2}$ causes fetal acidosis, yet the clinical relevance remains unknown; it can be partially addressed by maternal hyperventilation and gas processing. A systematic review of 11 fetoscopic spina bifida repair studies found no difference in the rate of ventriculoperitoneal shunting or hindbrain herniation reversal compared with open fetal surgery. Fetoscopy was associated with a higher rate of preterm prelabour rupture of the membranes (PPROM, $79 \%$ vs $36 \%$ ), preterm birth ( $96 \%$ vs $81 \%)$ and an increased need for additional neonatal surgery (30\% vs $7 \%)$. Uterine dehiscence did not occur in the fetoscopic group ( $0 \%$ vs $8 \%$ ), however the rate of placental abruption did not differ between the two groups. Therefore, fetoscopic repair does not yet clearly confer a fetal benefit equal to open repair and is associated with higher rates of preterm birth. Given the potential for reduction in maternal morbidity and the possibility of vaginal delivery, it is hoped that further technical development and experience will result in an optimal technique and reduce harm to the mother. 


\section{Practice points}

- In babies with open spina bifida, open fetal surgical repair prior to 26 weeks' gestation improves ventriculoperitoneal shunt rates and hindbrain herniation at 12 months of age and mobility at 30 months of age.

- The main fetal risk of surgery is preterm birth, with an average gestational age of 34 weeks after fetal surgery.

- Maternal risks include surgical complications, uterine dehiscence or rupture and the need for caesarean delivery in all pregnancies.

- Fetoscopic repair of spina bifida has the potential to reduce these maternal risks, although it currently carries a higher rate of prematurity and risk for postnatal neurosurgical intervention.

\section{Further reading}

Adzick NS, Thom EA, Spong CY et al. A randomized trial of prenatal versus postnatal repair of myelomeningocele. NEJM 2011;364:993-1004.

Sacco A, Van der Veeken L, Bagshaw E, et al. Maternal complications following open and fetoscopic fetal surgery: a systematic review and meta-analysis. PND 2019;39:251-68.

Inversetti A, Van Der Veeken L, Thompson D et al. Neurodevelopmental outcome in children with spina bifida aperta repaired prenatally versus postnatally: a systematic review and meta-analysis. UOG 2018.

Joyeux L, Danzer E, Flake A et al. Fetal surgery for spina bifida aperta. Arch Dis Child Fetal Neonatal Ed, 2018. 103(6): p. F589-F595. 
Kabagambe S, Jensen G, Chen YJ, Vanover MA, Farmer DL. Fetal surgery for myelomeingocele: a systematic review and meta-analysis of outcomes in fetoscopic versus open repair. FDT 2018;43:16174. 